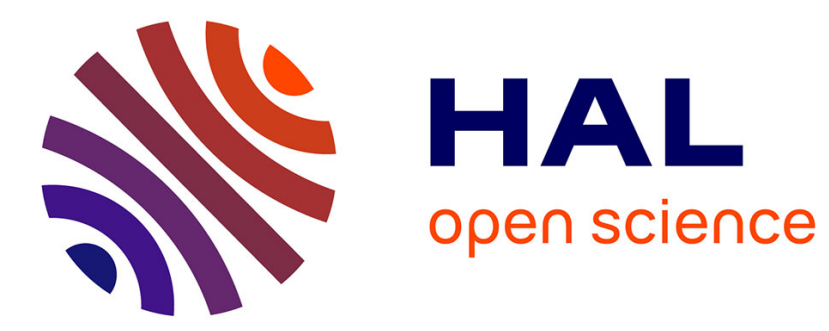

\title{
Generating chaotic attractors on a surface
}

Cristina Morel, Radu Vlad, Jean-Yves Morel, Dorin Petreus

\section{To cite this version:}

Cristina Morel, Radu Vlad, Jean-Yves Morel, Dorin Petreus. Generating chaotic attractors on a surface. Mathematics and Computers in Simulation, 2011, 81, pp.2549-2563. 10.1016/j.matcom.2011.05.003 . hal-01202688

\section{HAL Id: hal-01202688 \\ https://hal.science/hal-01202688}

Submitted on 24 Feb 2018

HAL is a multi-disciplinary open access archive for the deposit and dissemination of scientific research documents, whether they are published or not. The documents may come from teaching and research institutions in France or abroad, or from public or private research centers.
L'archive ouverte pluridisciplinaire HAL, est destinée au dépôt et à la diffusion de documents scientifiques de niveau recherche, publiés ou non, émanant des établissements d'enseignement et de recherche français ou étrangers, des laboratoires publics ou privés. 


\title{
Generating chaotic attractors on a surface
}

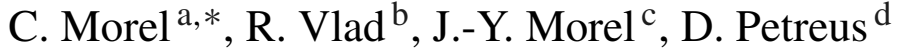 \\ a École Supérieure d'Électronique de l'Ouest - ESEO, 4 rue Merlet de la Boulaye, 49009 Angers, France \\ ${ }^{\mathrm{b}}$ Technical University of Cluj-Napoca, 103-105 Bd. Muncii, 400641 Cluj-Napoca, Romania \\ ${ }^{\mathrm{c}}$ University of Angers, 4 Bd. Lavoisier, 49100 Angers, France \\ ${ }^{\mathrm{d}}$ Technical University of Cluj-Napoca, 26-28 St. G. Baritiu, 400027 Cluj-Napoca, Romania
}

\begin{abstract}
The present paper introduces a new method to generate several independent periodic attractors, based on a switching piecewiseconstant controller. We demonstrate here that the state space equidistant repartition of these attractors is on an arbitrarily precise zone of a paraboloid or plane. We determine the state space domains where the attractors are generated from different initial conditions. A mathematical formula giving their maximal number in function of the controller piecewise-constant values is then deduced.
\end{abstract}

Keywords: Anticontrol of chaos; Independent chaotic attractors; Initial conditions; Switching piecewise-constant controller

\section{Introduction}

The study of chaotic dynamics has evolved from the traditional trend of understanding and analyzing chaos to the new attempt of utilizing it. Recently, there has been increasing interest in exploiting chaotic dynamics in engineering applications, such as telecommunications [1,8,14,25], information processing [13], electrical engineering [4], whereas much attention has focused on effectively generating chaos.

Creating chaos becomes a key in such applications where chaos is important and useful. It is well known that chaos can be generated via different approaches, such as linear feedback techniques or switching methods, to obtain various chaotic attractors $[3,11,12]$ from the new chaotic system $[12,23]$ or circuits $[22,26]$. Another technique to create chaos is to use a time-delay feedback perturbation on a system parameter or to employ an exogenous time-delay state-feedback input. This chaotification reference method designs a simple nonlinear feedback controller with an arbitrarily small amplitude leading to a chaotic dynamics in the controlled system [10,24].

The effect of time delay on the differential system can be observed in [19-21], with co-existing trivial attractors (fixed points or limit cycles) and chaotic attractors. A similar phenomenon generating various stable limit cycles is presented in [6,9]. In [9] it is proved that two sets of initial conditions produce two different stable limit cycles and a new limit cycle for each new initial condition selected is observed in [6].

\footnotetext{
* Corresponding author.

E-mail addresses: cristina.morel@eseo.fr (C. Morel), radu.constantin.vlad@mis.utcluj.ro (R. Vlad), jean-yves.morel@univ-angers.fr (J.-Y. Morel), dorin.petreus@ael.utcluj.ro (D. Petreus).
} 
Dynamical systems with a piecewise defined system function are a very important subject of interest. They occur naturally in many scientific disciplines. For instance, in electronics adequate models of some power circuits (such as $\mathrm{dc} / \mathrm{dc}$ converters) are represented by dynamical systems with piecewise-smooth system functions. The models of some mechanical systems [2] such as impact or stick-slip oscillators are represented by dynamical systems with piecewisesmooth discontinuous system functions. Recently, several types of border collision related bifurcations were found, like corner collision, sliding and grazing bifurcations [5][7].

After the study of the harmonics of chaotic systems $[16,18]$, we generated attractors using a switching piecewisebinary controller in a precise but uncontrollable area of the state space [15][17]. In this paper, we propose a switching piecewise-constant controller in continuous-time systems to generate several independent ${ }^{1}$ chaotic attractors in a controllable state space area. The attractors are reached from different initial conditions and are generated on a targeted zone of a precise curve situated on a paraboloid.

The generation of the attractors in the state space domain respects the few analysis steps of the nonlinear system. The first step is calculus of its equilibria. Their graphical representation in function of the parameter gives a precise curve in the state space. After that, it is necessary to determinate the parameter variation domain so that the equilibria of the nonlinear parameterized system are stable. The state space domains where the attractors are generated is obtained easily. The next step represents the replacement of the static variation of the parameter by a fast dynamics variation (a switching piecewise-constant controller) to generate several independent chaotic attractors around the stable equilibria. This controller represents a nonlinear feedback, as a sine function of the system state. The sine anticontrol feedback frequency imposes an attractor periodicity in the state space. Finally, the attractors maximal number in function of the piecewise-constant controller values and the sine anticontrol feedback frequency is calculated.

Throughout this study, the proposed methodology is illustrated with an example: the independent chaotic attractors are generated on two different paraboloids. In the second example, even if the system of differential equations is almost identical to the first one, the attractors are generated on two different planes.

Switch-mode power supplies usually emit electromagnetic interferences at the switching frequency and its harmonics. The application of this nonlinear feedback controller to switch-mode power supplies reduces the output voltage spectral emissions, and also maintains a small ripple in the output [16,18]. The design of this controller is based on the property that chaotified nonlinear systems present many independent chaotic attractors of small dimensions.

\section{Equilibria of a nonlinear system}

Consider a three-dimensional nonlinear autonomous system:

$$
\dot{n}=f_{p}(n), \quad n(0)=n_{0}, \quad t \in \mathbb{R}^{+}
$$

where $n=(x, y, z)^{T} \in \mathbb{R}^{3}, p \in \mathcal{P} \subseteq \mathbb{R}$ is a real parameter and $f_{p}: \mathbb{R}^{3} \rightarrow \mathbb{R}^{3}$ a nonlinear vector function.

The equilibria of the system (1) are found by solving the equations $f_{p}(n)=0$. The graphical representation of these equilibria in function of the parameter $p$ gives a precise curve in the state space $\mathbb{R}^{3}$. Let us take into account a variation of $p \in \mathcal{P}$, for which the equilibria are stable as follows:

$$
p_{a} \leq p \leq p_{b}
$$

We introduce the following notations: $\mathcal{X}$ the set of all equilibrium points, $\Gamma$ the set of all corresponding curves and $\Gamma_{X^{*}}$ the curve corresponding to the equilibrium point $X^{*} \in \mathcal{X}$.

Considering the Jacobian matrix for the equilibria and calculating its characteristic equation, we can investigate the stability of each equilibrium based on the Routh-Hurwitz conditions of the system characteristic equation. We determine the parameter variation domain such as the equilibria are stable.

Suppose that $X^{*}$ is stable for $p \in\left[p_{a}, p_{b}\right]$ and the projections of $\Gamma_{X^{*}}$ are the single intervals $\left[x_{a} ; x_{b}\right],\left[y_{a} ; y_{b}\right],\left[z_{a} ; z_{b}\right]$ (see Fig. 1).

In this paper, we are interested in generating several independent chaotic attractors on the 3D domain on some curve $\Gamma_{X^{*}}$, by switching the control parameter $p$ (between among the values $p_{a}$ and $p_{b}$ ).

\footnotetext{
${ }^{1}$ The generated attractors are disjuncts and the trajectory of an attractor will remain inside its own basin of attraction without switching to the basin of attraction of the neighboring attractors.
} 


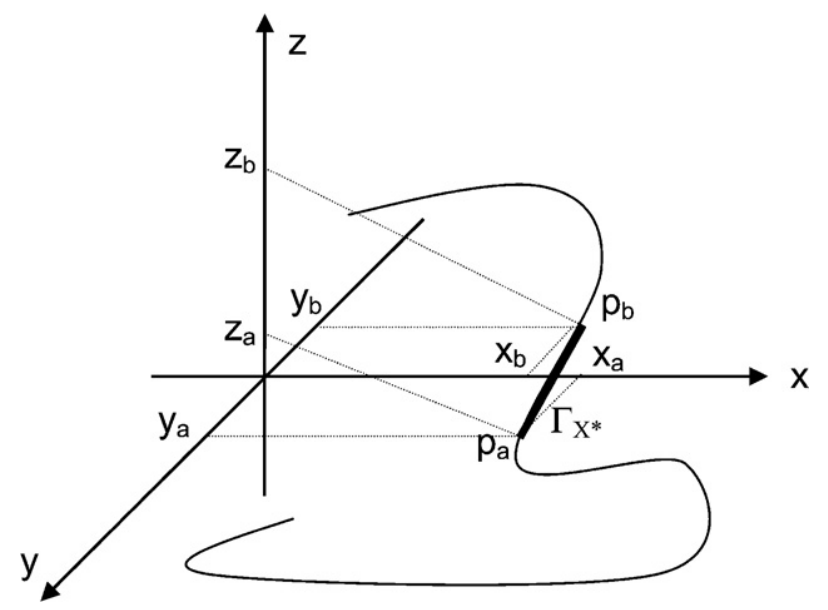

Fig. 1. Example of the system (1) equilibria on three-dimensional state space.

\section{Generating independent chaotic attractors in a nonlinear system}

Recently, in [19-21] a periodic nonlinearity of $p$ as $\mu \sin (x(t-\tau))\left[1+\epsilon \sin \left(x^{2}(t-\tau)\right)\right]$, has been considered. The global behavior of the delay model shows the co-existence of trivial attractors (fixed point or limit cycle) and chaotic attractors (with stable and unstable equilibria) generated in a precise state space for $\epsilon<1$ and $\mu \tau<\pi / 2$. With another nonlinear function $p, \epsilon \sin (\sigma x(t-\tau))$, Wang et al. [24] obtains only two separated chaotic attractors near two stable fixed points for large values of $\sigma$.

In order to generate independent chaotic attractors for the system (1), let us introduce a piecewise-constant characteristic of the feedback controller $p$, defined analytically as follows:

$$
p= \begin{cases}p_{b}, & g(t)<u(x(t), y(t), z(t)) \\ p_{a}, & g(t) \geq u(x(t), y(t), z(t)) \quad \text { for } t \geq 0\end{cases}
$$

where $g$ is a periodic function and $u$ the anticontrol state feedback. We propose to use the anticontrol with a piecewiseconstant controller, hereafter called anticontrol switching piecewise-constant controller. The application of the classical anticontrol of chaos introduced in [24] (see also e.g. [10,16]) uses a nonlinear state feedback. In this paper we choose:

$$
u(x(t), y(t), z(t))=\varepsilon \sin (\sigma x(t)),
$$

as in [15,24], while for $g$

$$
g(t)=\sin (\delta t),
$$

as in Fig. 2. The fast switchings of the controller between the levels $p_{a}$ and $p_{b}$, determine an attraction of the state variable $x$ first to $x_{a}$ and then to $x_{b}$, and so on. The size of the attractors obtained are rather small (see Fig. 3).

The dynamical state space trajectory remains around the equilibrium point, for each attractor starting from different initial conditions. With the anticontrol switching piecewise-constant controller of Eq. (3), the attractors will be chaotic along $\Gamma_{X^{*}}$ for $p \in\left[p_{a}, p_{b}\right]$ (see the tick line in Fig. 1).

The study of the chaotic attractors shows an equidistant repartition in the state space. Fig. 3 shows two transient state space trajectories until two independent chaotic attractors are reached.

The transitions $p_{a} \rightarrow p_{b} \rightarrow p_{a} \rightarrow p_{b}$ of the anticontrol switching piecewise-constant controller $p$ of Eq. (3) determine a broken-line characteristic of the transient state space trajectory. The times $\left(t_{k}\right)_{k \in \mathbb{N}}$ of the transitions $p_{b} \rightarrow p_{a}$ of $p$ are symbolized by circles $\bullet$ on the state space. At each time $\left(t_{k}\right)_{k \in \mathbb{N}}, g(t)$ is equal to $u(x(t), y(t), z(t))$. Consequently, we can write:

$$
g(t)=u(x(t), y(t), z(t)) .
$$




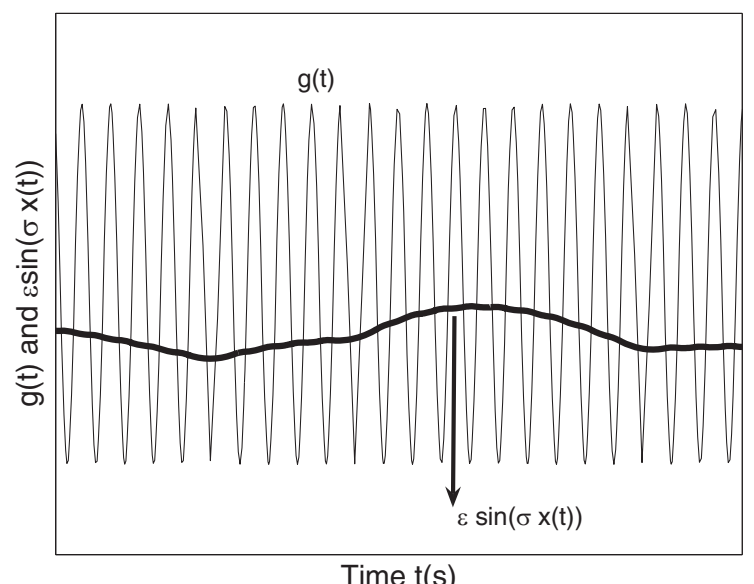

Fig. 2. Fast dynamics of $g(t)$ : example of sinus function $g$ and the anticontrol of chaos state feedback $u$.

According to Eq. (4), Eq. (6) becomes:

$$
g(t)=\varepsilon \sin (\sigma x(t)) .
$$

For $t=t_{0}$, the solution of Eq. (7) is:

$$
x\left(t_{0}\right)=\frac{1}{\sigma} \arcsin \frac{g\left(t_{0}\right)}{\varepsilon} .
$$

The $2 \pi$ periodicity of the sine function of $u(t)$ enables to find all the solutions $x\left(t_{k}\right)$ of Eq. (6):

$$
\sigma x\left(t_{k}\right) \pm 2 k \pi=\arcsin \frac{g\left(t_{0}\right)}{\varepsilon}, \quad k \in \mathbb{N} .
$$

Then,

$$
x\left(t_{k}\right)=\frac{1}{\sigma} \arcsin \frac{g\left(t_{0}\right)}{\varepsilon} \pm \frac{2 k \pi}{\sigma}, \quad k \in \mathbb{N} .
$$

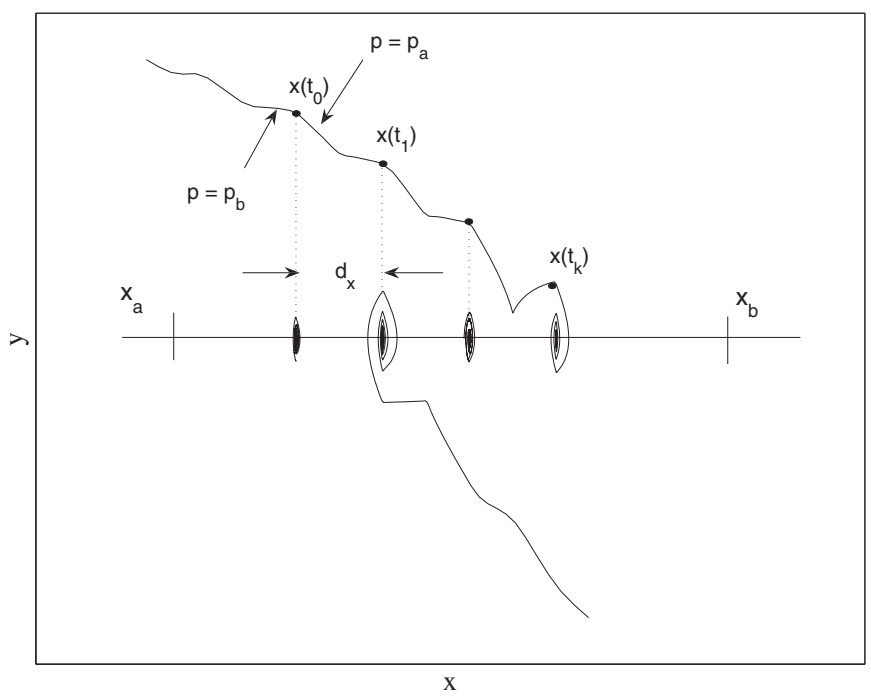

Fig. 3. Example of independent chaotic attractors ( $x-y$ phase projection). 

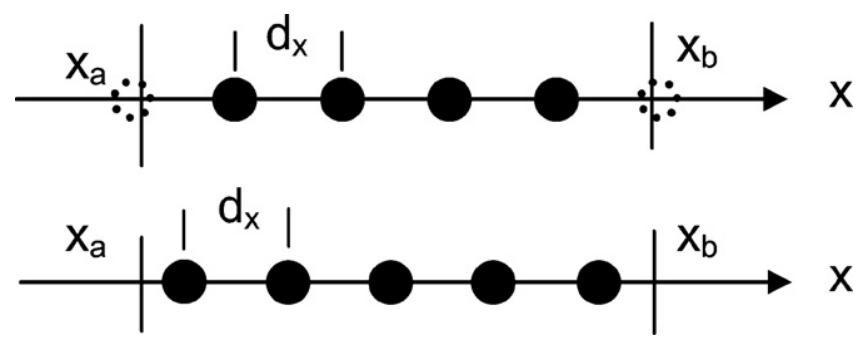

Fig. 4. Two possible attractors repartition inside the state space domain $\left[x_{a} ; x_{b}\right]$.

We can also write:

$$
x\left(t_{k}\right)-x\left(t_{k-1}\right)=\frac{2 \pi}{\sigma}, \quad k \in N^{+} .
$$

We have demonstrated that the periodicity of the $x(t)$ function is $2 \pi / \sigma$, i.e. the distance on the $x$-axis between two consecutive transitions $p_{b} \rightarrow p_{a}$ of $p$. Therefore, after many numerical simulations, we have deduced that the distance between two consecutive attractors on the $x$-axis coincides with the distance between two circles $\bullet$ on the $x$-axis. The attractors periodicity in the state space depends on the sine anticontrol feedback $u(x(t), y(t), z(t))$ frequency given by the following relation:

$$
d_{x}=\frac{2 \pi}{\sigma} .
$$

The $d_{x}$ periodicity of attractors on the $x$-axis enables to find the maximal number of the attractors generated on the bold curve of Fig. 1, inside the $x$ state space domain $\left[x_{a} ; x_{b}\right]$ :

$$
N_{\max }=\left[\frac{x_{b}-x_{a}}{d_{x}}\right]+1
$$

$N_{\max }$ of Eq. (13) gives the theoretical number of attractors that can be generated. The position of the first attractor generated inside the $x$ state space domain $\left[x_{a} ; x_{b}\right]$ (near $x_{a}$ ) is given by $X^{*}$, which depends on the parameters system of Eq. (1). The following attractors are equidistant, separated by a $d_{x}$ distance. Two possible cases of repartition of chaotic attractors are given in Fig. 4. A difference of one attractor may sometimes occur between the theoretical and the simulated attractors number as discussed in the next section.

\section{Attractor generation on the paraboloid}

In order to illustrate the proposed approach, we consider a system dynamics described by the following parameterized equations:

$$
\left\{\begin{array}{l}
\dot{x}=\alpha(y \cos \theta-x \sin \theta) \\
\dot{y}=x \sin \theta-y \cos \theta-x z+p \\
\dot{z}=x^{2}+y^{2}-\beta z
\end{array}\right.
$$

which are in the form (1). When $p=0, \alpha=10, \beta=8 / 3$ and $\theta=45^{\circ}$, the system (14) is not chaotic and, in fact, has the origin as a globally exponentially stable equilibrium point. The Jacobian is given by

$$
J_{x, y, z}=\left[\begin{array}{ccc}
-\alpha \sin \theta & \alpha \cos \theta & 0 \\
\sin \theta-z & -\cos \theta & -x \\
2 x & 2 y & -\beta
\end{array}\right]
$$


Obviously, $X^{*}=(0,0,0)$ is a trivial equilibrium. Linearizing the system (14) around the equilibrium $X^{*}$ provides three eigenvalues: $\lambda=0,-\beta,-\cos \theta-\alpha \sin \theta$. The system has marginal stability. To find a nontrivial equilibrium point, we may observe that the first equation in (14) yields to

$$
y=x \frac{\sin \theta}{\cos \theta} .
$$

So that the third one gives

$$
z=\frac{x^{2}+y^{2}}{\beta}=\frac{x^{2}}{\beta \cos ^{2} \theta}
$$

and the second one gives

$$
p=x z=\frac{x^{3}}{\beta \cos ^{2} \theta} .
$$

The system (14) has one equilibrium point depending on $p$. Thus:

$$
X^{*}=\left(\sqrt[3]{p \beta \cos ^{2} \theta}, \sin \theta \sqrt[3]{\frac{p \beta}{\cos \theta}}, \sqrt[3]{\frac{p^{2}}{\beta \cos ^{2} \theta}}\right)
$$

This time linearizing the system (14) around the equilibrium point $X^{*}$ yields the following Jacobian matrix and its characteristic equation:

$$
\lambda I-J_{X^{*}}=\left[\begin{array}{ccc}
\lambda+\alpha \sin \theta & -\alpha \cos \theta & 0 \\
\sqrt[3]{\frac{p^{2}}{\beta \cos ^{2} \theta}-\sin \theta} & \lambda+\cos \theta & \sqrt[3]{p \beta \cos ^{2} \theta} \\
-2 \sqrt[3]{p \beta \cos ^{2} \theta} & -2 \sin \theta \sqrt[3]{\frac{p \beta}{\cos \theta}} & \lambda+\beta
\end{array}\right]
$$

and with the characteristic polynomial

$$
\begin{aligned}
P(\lambda)= & \lambda^{3}+(\beta+\cos \theta+\alpha \sin \theta) \lambda^{2}+\left[\beta(\cos \theta+\alpha \sin \theta)+\sqrt[3]{p^{2} \beta^{2} \cos \theta}\left(2 \sin \theta+\frac{\alpha}{\beta}\right)\right] \lambda \\
& +3 \alpha \sqrt[3]{p^{2} \beta^{2} \cos \theta} .
\end{aligned}
$$

Let us apply now the Routh-Hurwitz Criterion. As known, a necessary and sufficient condition for the stability of the equilibrium point $X^{*}$ is that the real parts of the roots $\lambda$ be negative, which can be confirmed if and only if the three following conditions hold:

$$
\begin{aligned}
& \beta+\cos \theta+\alpha \sin \theta>0, \\
& 3 \alpha \sqrt[3]{p^{2} \beta^{2} \cos \theta}>0, \\
& -3 \alpha \sqrt[3]{p^{2} \beta^{2} \cos \theta}+(\beta+\cos \theta+\alpha \sin \theta) \cdot\left[\beta(\cos \theta+\alpha \sin \theta)+\sqrt[3]{p^{2} \beta^{2} \cos \theta}\left(2 \sin \theta+\frac{\alpha}{\beta}\right)\right]>0 .
\end{aligned}
$$

A variation of $z$ between

$$
z \in\left[z_{a} ; z_{b}\right]
$$

imposes the intervals to the equilibrium $X^{*}$ on the state space. The state space for the $x$-axis projection according to Eq. (17) is

$$
x \in\left[\sqrt{z_{a} \beta \cos ^{2} \theta} ; \sqrt{z_{b} \beta \cos ^{2} \theta}\right],
$$


Table 1

The system (14): $\alpha=10, \sigma=100$ and $\varepsilon=5$.

\begin{tabular}{llllllllllll}
\hline Curve & $\beta$ & $\theta$ & Eq. (22) & Eq. (23) & Eq. (24) & $z_{a}$ & $z_{b}$ & $\begin{array}{l}x \\
\text { Eq. (26) }\end{array}$ & $\begin{array}{l}y \\
\text { Eq. (27) }\end{array}$ & $\begin{array}{l}p \\
\text { Eq. (28) or (29) }\end{array}$ \\
\hline 1 & & $\pi / 6$ & 8.5 & {$[6.9,41.5]$} & {$[135,148]$} & 0.1 & 0.6 & {$[0.45,1.09]$} & {$[0.26,0.63]$} & {$[0.04,0.66]$} & 10 \\
2 & $8 / 3$ & $5 \pi / 12$ & 12.6 & {$[0,14.5]$} & {$[333,353]$} & 0 & 0.7 & {$[0,0.35]$} & {$[0,1.32]$} & {$[0,0.247]$} \\
3 & & $4 \pi / 3$ & 11.8 & {$[4,30]$} & {$[293,323]$} & 0.1 & 0.75 & {$[-0.71,-0.26]$} & {$[-1.22,-0.45]$} & {$[-0.53,-0.026]$} & 8 \\
4 & & $\pi / 3$ & 15.8 & {$[10,50]$} & {$[973,1001]$} & 0.1 & 0.5 & {$[0.4,0.91]$} & {$[0.707,1.58]$} & {$[0.04,0.45]$} & 9 \\
5 & $20 / 3$ & $7 \pi / 6$ & 12.5 & {$[34.6,86.6]$} & {$[491,493]$} & 0.2 & 0.5 & {$[-1.58,-1]$} & {$[-0.91,-0.57]$} & {$[-0.79,-0.2]$} & 10 \\
6 & & $17 \pi / 12$ & 16.6 & {$[0,25.8]$} & {$[1096,1119]$} & 0 & 0.5 & {$[-0.47,0]$} & {$[-1.76,0]$} & {$[-0.434,0]$} & 8 \\
\hline
\end{tabular}

or

$$
x \in\left[-\sqrt{z_{b} \beta \cos ^{2} \theta} ;-\sqrt{z_{a} \beta \cos ^{2} \theta}\right] .
$$

According to Eq. (16), the state space for the $y$-axis projection has the following form:

$$
y \in\left[\sqrt{z_{a} \beta \sin ^{2} \theta} ; \sqrt{z_{b} \beta \sin ^{2} \theta}\right]
$$

or

$$
y \in\left[-\sqrt{z_{b} \beta \sin ^{2} \theta} ;-\sqrt{z_{a} \beta \sin ^{2} \theta}\right] .
$$

The switch levels of the anticontrol switching piecewise-constant controller $p$ of Eq. (18) is determinated as follow:

$$
p \in\left[z_{a} \sqrt{z_{a} \beta \cos ^{2} \theta} ; z_{b} \sqrt{z_{b} \beta \cos ^{2} \theta}\right]
$$

or

$$
p \in\left[-z_{b} \sqrt{z_{b} \beta \cos ^{2} \theta} ;-z_{a} \sqrt{z_{a} \beta \cos ^{2} \theta}\right] .
$$

So if the anticontrol $p$ has the form of Eq. (28), the attractors are generated on the curve $\Gamma_{X^{*}}$ having the projections

$$
\left\{x \in\left[\sqrt{z_{a} \beta \cos ^{2} \theta} ; \sqrt{z_{b} \beta \cos ^{2} \theta}\right], y \in\left[\sqrt{z_{a} \beta \sin ^{2} \theta} ; \sqrt{z_{b} \beta \sin ^{2} \theta}\right], z \in\left[z_{a} ; z_{b}\right]\right\},
$$

On the other hand, with the anticontrol switching piecewise-constant controller of Eq. (29), the attractors are generated on the domain:

$$
\left\{x \in\left[-\sqrt{z_{b} \beta \cos ^{2} \theta} ;-\sqrt{z_{a} \beta \cos ^{2} \theta}\right], y \in\left[-\sqrt{z_{b} \beta \sin ^{2} \theta} ;-\sqrt{z_{a} \beta \sin ^{2} \theta}\right], z \in\left[z_{a} ; z_{b}\right]\right\}
$$

Considering a fast dynamic variation of $p$ given by Eq. (3), the independent chaotic attractors are generated inside the state space of Eq. (30) or (31). Taking into account the distance between two consecutive attractors on the $x$-axis given by Eq. (12), the maximum number of independent chaotic attractors of Eq. (13) generated on the interval of Eq. (30) or (31) is given by:

$$
N_{\max }=\left[\frac{\sigma \sqrt{\beta \cos ^{2} \theta}}{2 \pi}\left(\sqrt{z_{b}}-\sqrt{z_{a}}\right)\right]+1
$$

In our simulations, $\alpha=10, \sigma=100$ and $\varepsilon=5$. With this values, the conditions (22)-(24) are verified. Therefore the equilibrium point $X^{*}$ of the system of Eq. (14) is stable. We list in Table 1 the equilibria intervals on each axis $x$, $y$ and $z$ (derived from Eqs. (25)-(27)) for six different values of $z_{a}$ and $z_{b}$. This table includes the maximum number of chaotic attractors of Eq. (32).

Let us see now if the results of the numerical simulations are in concordance with those given in Table 1 by the mathematical formulas (30) or (31) and (32). Fig. 5 shows the independent chaotic attractors of the system (14). The attractors, generated from different initial conditions, are situated in the plane $y=x(\sin \theta / \cos \theta)$, and on the parabola $z=\left(x^{2}+y^{2}\right) / \beta$ represented in Fig. 5 with the fine curve. The independent attractors are generated on this fine curve on 


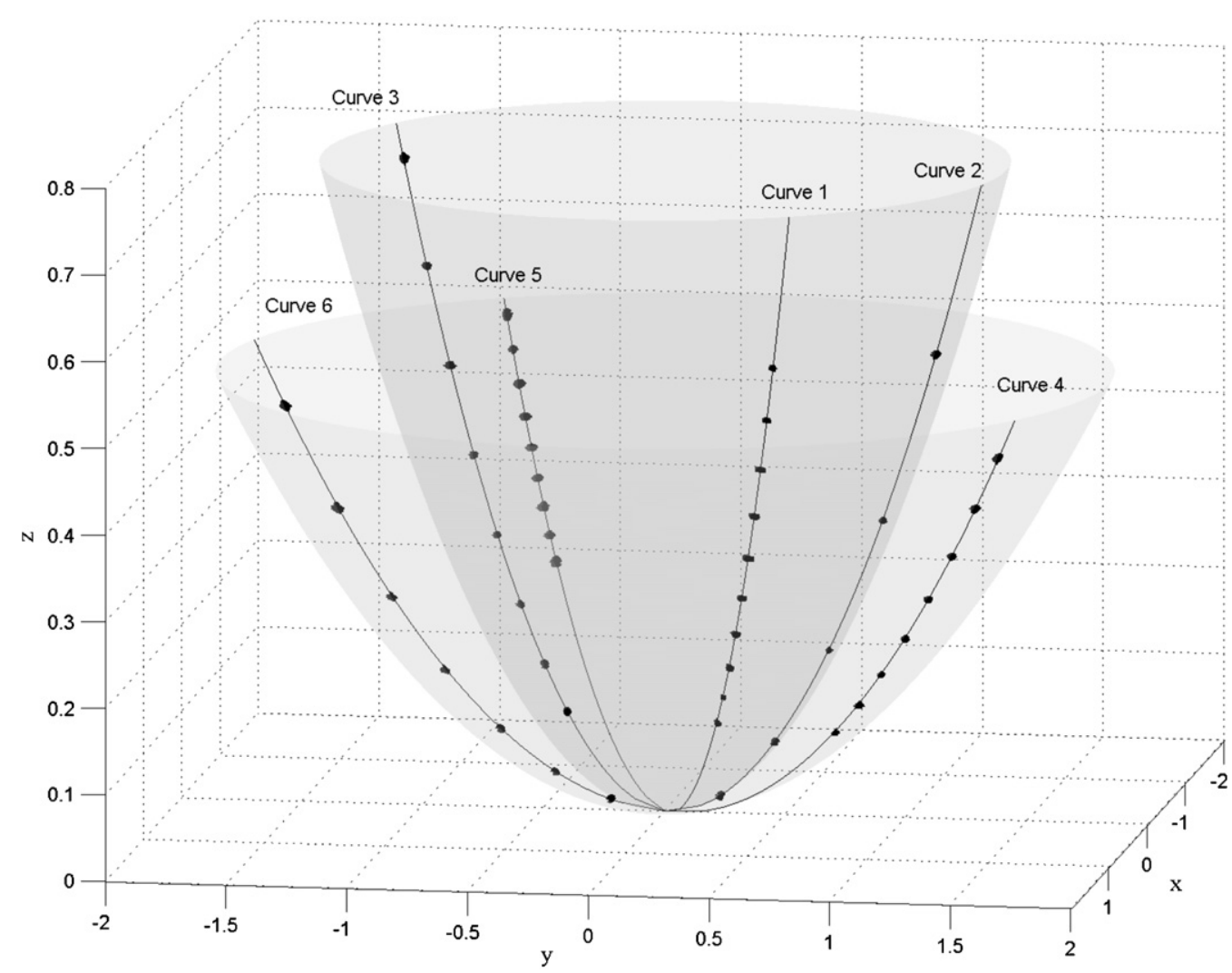

Fig. 5. The independent chaotic attractors generation of the system (14) on the curves 1, 2 and 3 of the first paraboloid. The independent chaotic attractors generation of the system (14) on the curves 4,5 and 6 of the second paraboloid.

a precise zone according to the $z_{a}$ and $z_{b}$ values of $z$ (as for curve 1). With a variation of $\theta$, the attractors are situated on other curves (curve 2 or curve 3 ). In the 3D state space, the curves are situated on the first paraboloid and the attractors also. Generating attractors to a different paraboloid is possible according to $\beta$. Figs. 6-11 present the projections of the generated attractors onto $x-z$ and $y-z$ planes. For the six intervals of $z$, all attractors are situated inside the state space
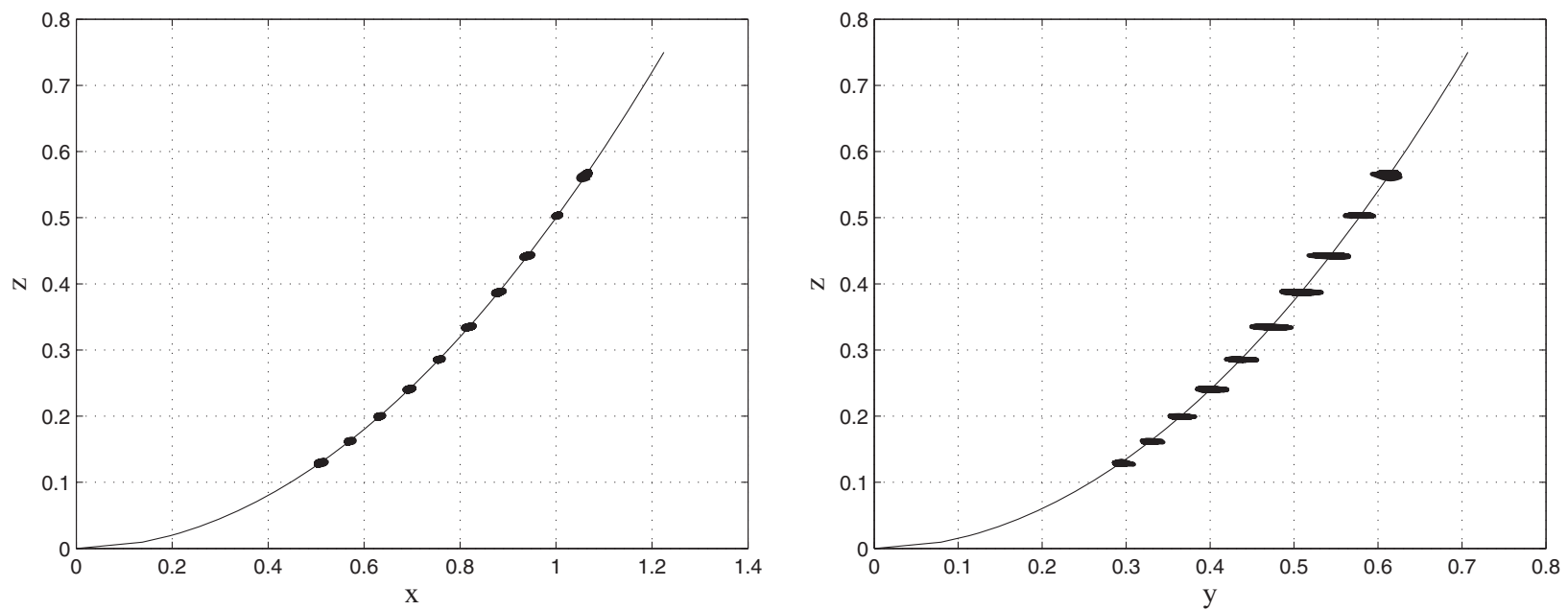

Fig. 6. The ten attractors projection onto $x-z$ and $y-z$ planes of the curve 1 , with the theoretical state space domain $x \in[0.45,1.09], y \in[0.26,0.63]$ and $z \in[0.1,0.6]$. 

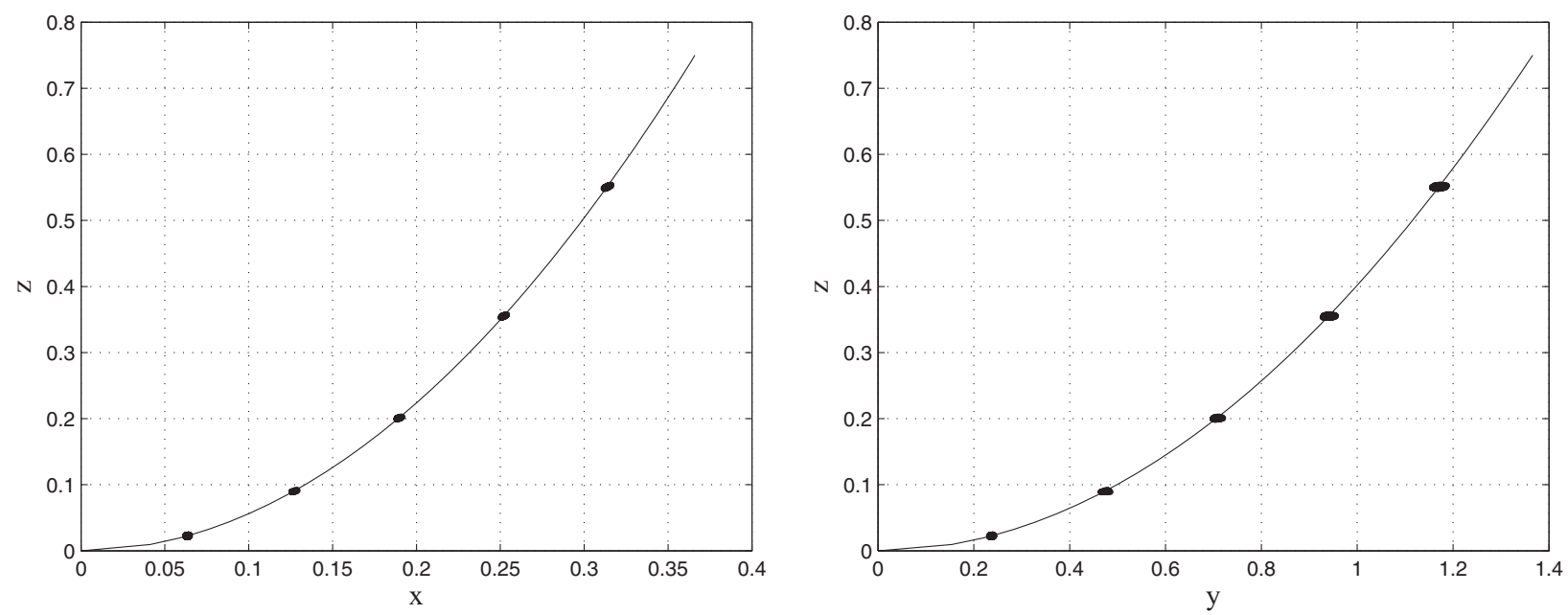

Fig. 7. The five attractors projection onto $x-z$ and $y-z$ planes of the curve 2 , with the theoretical state space domain $x \in[0,0.35], y \in[0,1.32]$ and $z \in[0,0.7]$.

given by Table 1 ( 9 and 10 columns). On the other hand, there is a difference of one attractor between the numerical simulation of the independent chaotic attractors number generated on the interval of Eq. (25) and the maximum number of attractors given by the mathematical formula of Eq. (32). For six values of the $z$ state space (columns 7 and 8 of Table 1), Fig. 5 presents six groups of independent chaotic attractors. The first case of attractors repartition inside the state space domain $z \in\left[z_{a} ; z_{b}\right]$ of the curves $2,4,5$ and 6 is available. This is why there is a difference of one attractor between the numerical simulation of the independent chaotic attractors number generated and the maximum attractors number given by Eq. (32). For the two other $z$ state space domains of curves 1 and 3, the theoretical and simulated attractors number coincide.

Fig. 12 presents the projections of one attractor onto $x-z$ plane. This attractor reaches the regime of operation $x=0.6$, $y=0.6$ and $z=0.135$.

The positive largest Lyapunov exponent has become the standard characteristics of a chaotic system. At the end of the present section, we introduce the Lyapunov exponent as a simple measure of sensitive dependence on initial conditions, distinguishing a chaotic from a nonchaotic trajectory by its positive value.

For a linear increasing variation of $\varepsilon$, the Lyapunov exponent has positive values as in Fig. 12, decreasing to negative values. The Lyapunov exponent of system (14) is calculated for distinct initial conditions (almost identical)
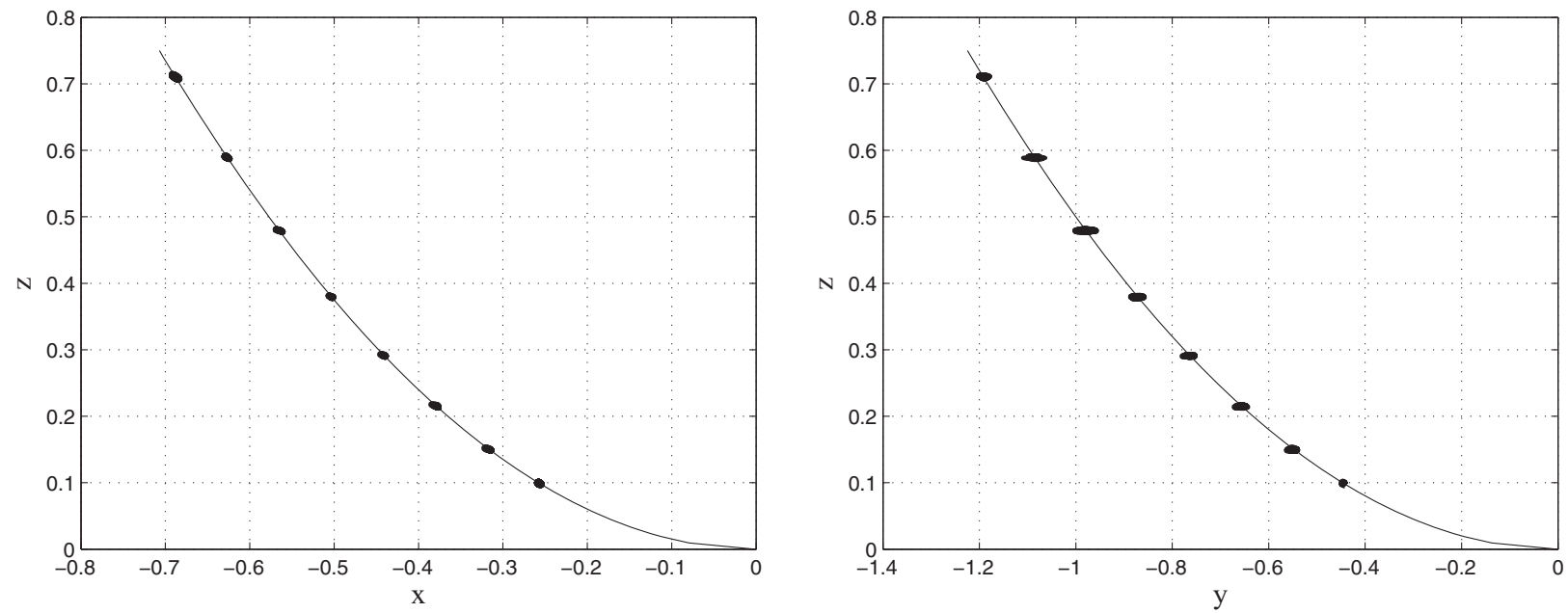

Fig. 8. The eight attractors projection onto $x-z$ and $y-z$ planes of the curve 3 , with the theoretical state space domain $x \in[-0.71,-0.26], y \in[-1.22$, $-0.45]$ and $z \in[0.1,0.75]$ 

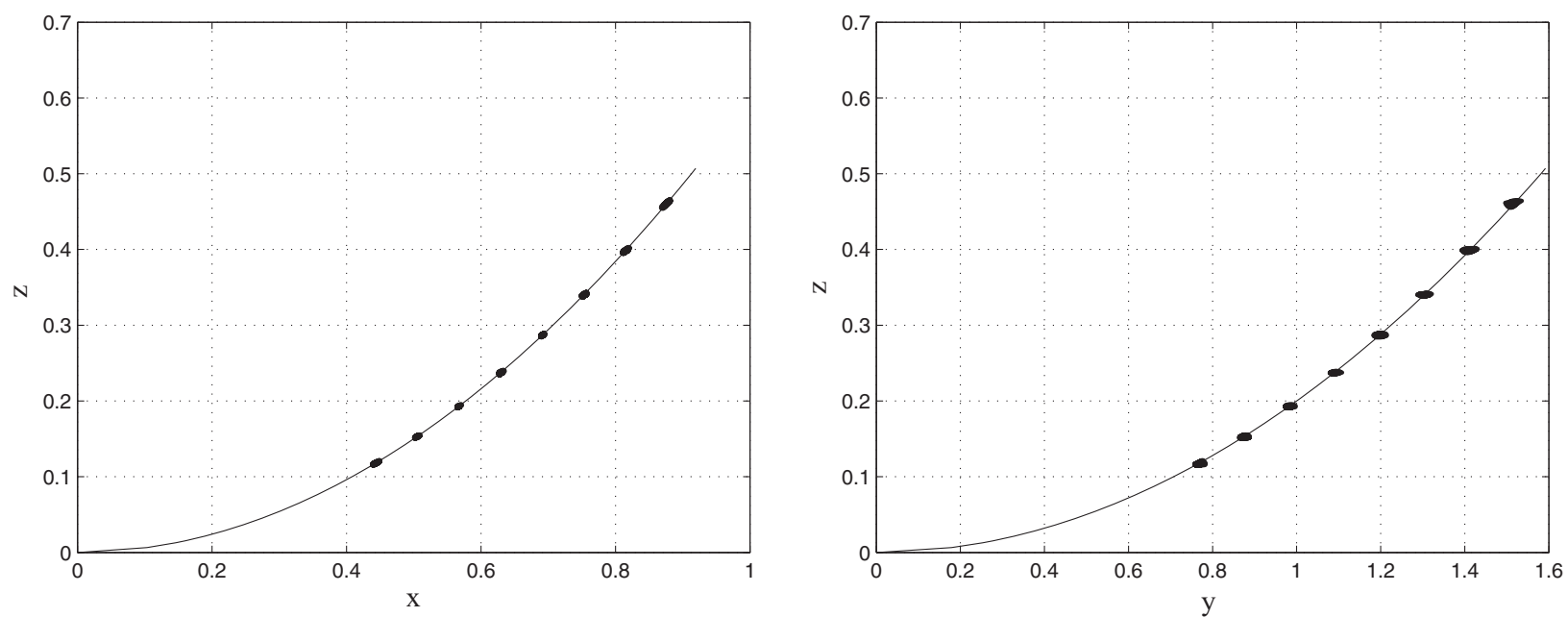

Fig. 9. The eight attractors projection onto $x-z$ and $y-z$ planes of the curve 4 , with the theoretical state space domain $x \in[0.4,0.91], y \in[0.707,1.58]$ and $z \in[0.1,0.5]$.

near an equilibrium point (i.e. near the basin of attraction). With almost identical initial conditions, but far away of the equilibrium points (outside of the basin of attraction), the system (14) can reach different regimes of operation (two different attractors), as in Fig. 13. This choice of initial conditions is a source of errors in the calculation of the Lyapunov exponent.

In this section, we show that the switching piecewise-constant controller of Eqs. (3)-(5) can drive the system (14) from nonchaotic (with zero equilibrium point) to chaotic (with several equilibria points) behaviors.

\section{Attractor generation on the plane}

In this section, we generate independent chaotic attractors from the following system:

$$
\left\{\begin{array}{l}
\dot{x}=\alpha(y \cos \theta-x \sin \theta), \\
\dot{y}=\gamma x \sin \theta-y \cos \theta-x z, \\
\dot{z}=x^{2}+y^{2}-\beta z+p,
\end{array}\right.
$$
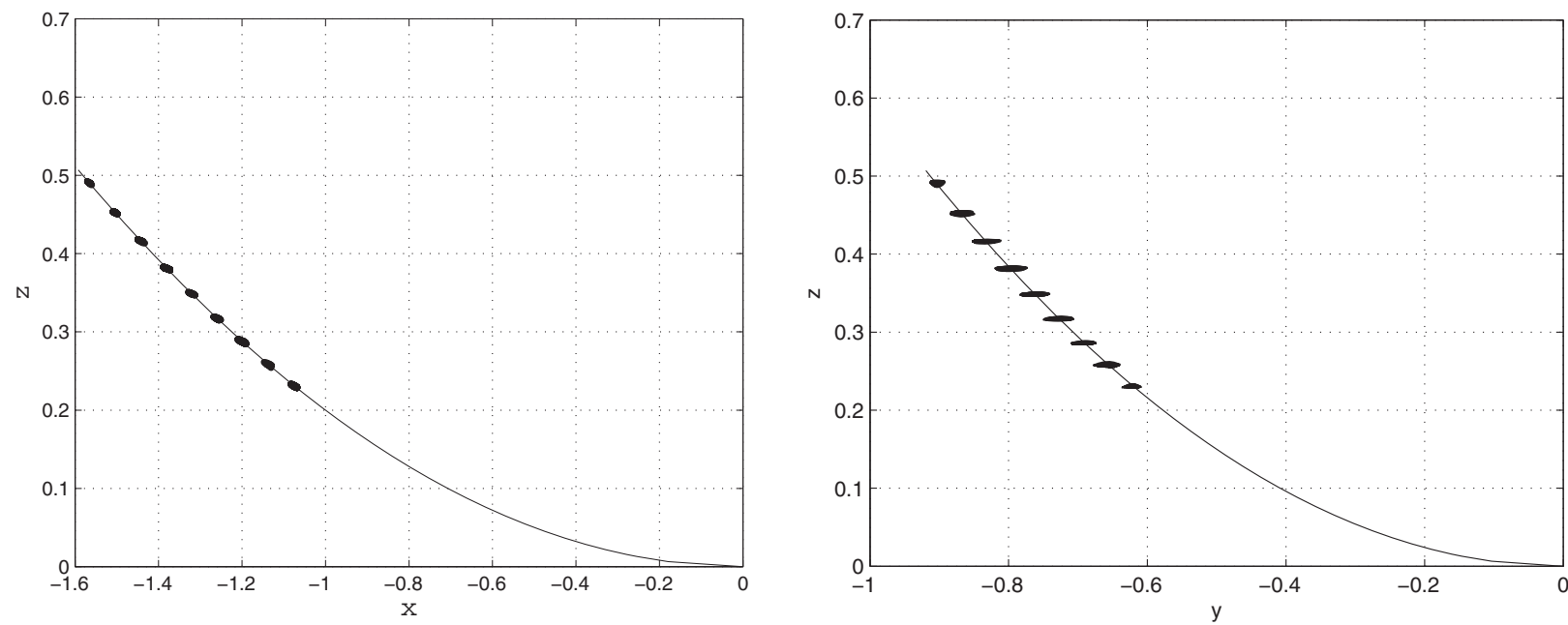

Fig. 10. The ten attractors projection onto $x-z$ and $y-z$ planes of the curve 5 , with the theoretical state space domain $x \in[-1.58,-1], y \in[-0.91$, $-0.57]$ and $z \in[0.2,0.5]$. 

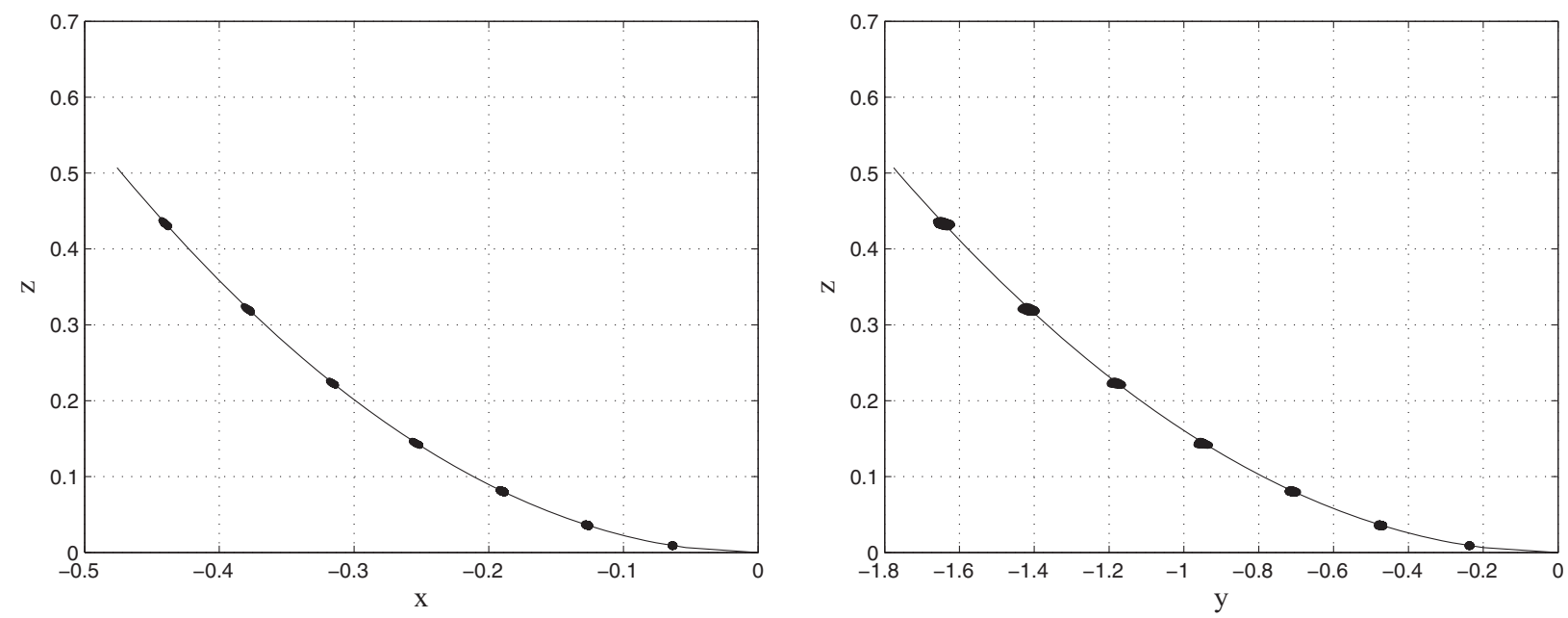

Fig. 11. The seven attractors projection onto $x-z$ and $y-z$ planes of the curve 6 , with the theoretical state space domain $x \in[-0.47,0], y \in[-1.76$, $0]$ and $z \in[0,0.5]$.

The corresponding Jacobian matrix is

$$
J_{x, y, z}=\left[\begin{array}{ccc}
-\alpha \sin \theta & \alpha \cos \theta & 0 \\
\gamma \sin \theta-z & -\cos \theta & -x \\
2 x & 2 y & -\beta
\end{array}\right]
$$

The equilibria of the system (33) are found by solving the equations $\dot{x}=\dot{y}=\dot{z}=0$, which leads to:

$$
\left\{\begin{array}{l}
\alpha(y \cos \theta-x \sin \theta)=0 \\
\gamma x \sin \theta-y \cos \theta-x z=0 \\
x^{2}+y^{2}-\beta z+p=0
\end{array}\right.
$$

Eq. (35) can then be written as:

$$
\begin{aligned}
& y=x \frac{\sin \theta}{\cos \theta}, \\
& z=(\gamma-1) \sin \theta,
\end{aligned}
$$
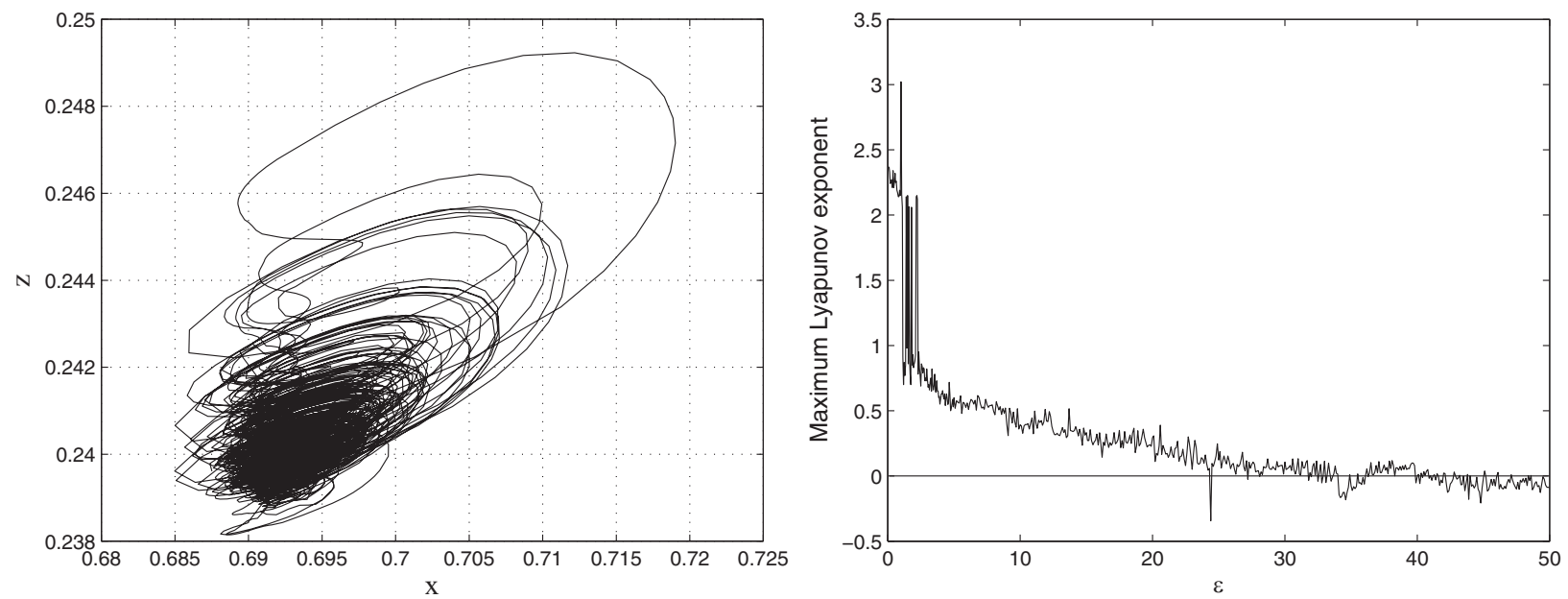

Fig. 12. A chaotic attractor projection of the system (14) onto $x-y$ plane. The largest Lyapunov exponent of the same system. 


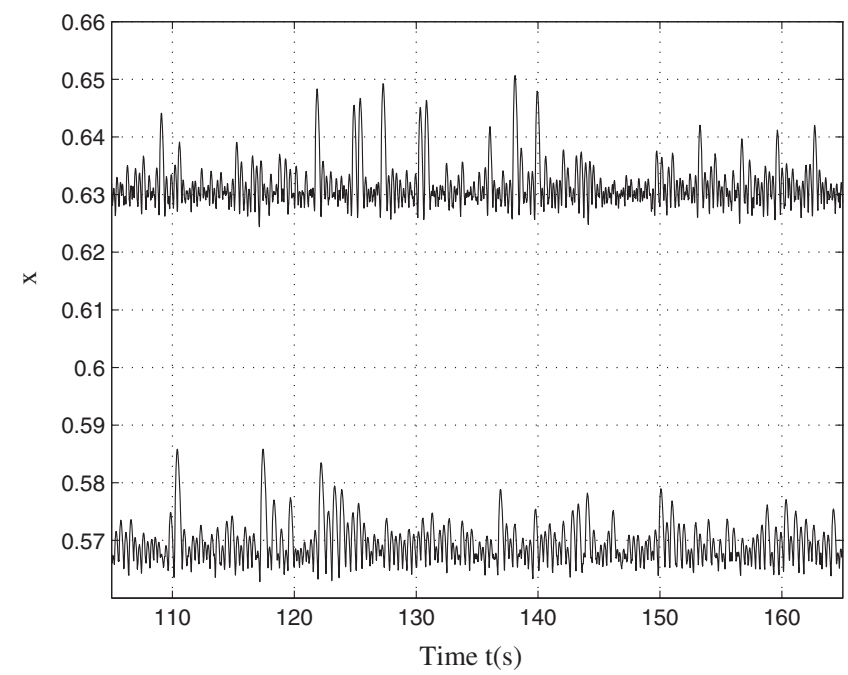

Fig. 13. The time waveform of the $x$ state variable of the system (14) starting from distinct initial conditions (almost identical) far away of the equilibrium points.

$$
p=\beta(\gamma-1) \sin \theta-x^{2} \frac{1}{\cos ^{2} \theta} .
$$

From the last Eq. (38), $x$ can be expressed as

$$
x^{2}=[\beta(\gamma-1) \sin \theta-p] \cos ^{2} \theta .
$$

So

$$
x= \pm|\cos \theta| \sqrt{\beta(\gamma-1) \sin \theta-p}
$$

and

$$
y= \pm \frac{\sin \theta}{\cos \theta}|\cos \theta| \sqrt{\beta(\gamma-1) \sin \theta-p} .
$$

The system of Eq. (33) has two equilibria $X_{+}^{*}$ and $X_{-}^{*}$, symmetrically placed with respect to the $x$ and $y$ axes:

$$
\begin{aligned}
& X_{+}^{*}=\left(|\cos \theta| \sqrt{\beta(\gamma-1) \sin \theta-p}, \frac{\sin \theta}{\cos \theta}|\cos \theta| \sqrt{\beta(\gamma-1) \sin \theta-p},(\gamma-1) \sin \theta\right) \\
& X_{-}^{*}=\left(-|\cos \theta| \sqrt{\beta(\gamma-1) \sin \theta-p},-\frac{\sin \theta}{\cos \theta}|\cos \theta| \sqrt{\beta(\gamma-1) \sin \theta-p},(\gamma-1) \sin \theta\right)
\end{aligned}
$$

Considering the Jacobian matrix for these equilibria and calculating its characteristic equation $\lambda I-J_{X_{ \pm}^{*}}$,

$$
\left[\begin{array}{ccc}
\lambda+\alpha \sin \theta & -\alpha \cos \theta & 0 \\
-\sin \theta & \lambda+\cos \theta & \pm|\cos \theta| \sqrt{\beta(\gamma-1) \sin \theta-p} \\
\mp 2|\cos \theta| \sqrt{\beta(\gamma-1) \sin \theta-p} & \mp 2 \frac{\sin \theta}{\cos \theta}|\cos \theta| \sqrt{\beta(\gamma-1) \sin \theta-p} & \lambda+\beta
\end{array}\right]
$$

We can investigate the stability of this equilibrium based on the Routh-Hurwitz conditions on system (33) characteristic equation. Therefore:

$$
\begin{aligned}
P(\lambda)= & \lambda^{3}+(\beta+\cos \theta+\alpha \sin \theta) \lambda^{2}+\left[\beta \cos \theta+\alpha \beta \sin \theta-2 p \sin \theta \cos \theta+2 \beta(\gamma-1) \sin ^{2} \theta \cos \theta\right] \lambda \\
& +2 \alpha \cos \theta[\beta(\gamma-1) \sin \theta-p] .
\end{aligned}
$$


Table 2

The system (33): $\alpha=10$.

\begin{tabular}{|c|c|c|c|c|c|c|c|c|c|c|c|}
\hline Line & $\begin{array}{l}\gamma \\
\text { Eq. (52) }\end{array}$ & $\theta$ & Eq. (46) & Eq. (47) & Eq. (48) & $x_{a}$ & $x_{b}$ & $\begin{array}{l}y \\
\text { Eq. (50) }\end{array}$ & $z$ & $\begin{array}{l}p \\
\text { Eq. (51) }\end{array}$ & $\begin{array}{l}N_{\max } \\
\text { Eq. (53) }\end{array}$ \\
\hline 1 & 3 & $\pi / 6$ & 8.5 & {$[0,6.4]$} & {$[129,133]$} & 0 & 0.5 & {$[0,0.28]$} & 1 & {$[2.33,2.66]$} & 8 \\
\hline 2 & 2.03 & $5 \pi / 12$ & 12.6 & {$[0,5.75]$} & {$[332.8,333]$} & 0 & 0.25 & {$[0,0.933]$} & 1 & {$[1.73,2.66]$} & 4 \\
\hline 3 & 2.15 & $4 \pi / 3$ & 11.8 & {$[2,12.5]$} & {$[287,288]$} & -0.5 & -0.2 & {$[-0.87,-0.35]$} & 1 & {$[1.66,2.506]$} & 5 \\
\hline 4 & -0.154 & $\pi / 3$ & 11.8 & {$[0.5,8]$} & {$[287,288]$} & 0.1 & 0.4 & {$[0.17,0.69]$} & -1 & {$[-3.306,-2.706]$} & 5 \\
\hline 5 & -1 & $7 \pi / 6$ & 8.5 & {$[4.1,16.5]$} & {$[123,131]$} & -0.8 & -0.4 & {$[-0.46,-0.23]$} & -1 & {$[-3.52,-2.88]$} & 7 \\
\hline 6 & -0.035 & $17 \pi / 12$ & 12.58 & {$[0,5.75]$} & {$[332.8,333]$} & -0.25 & 0 & {$[-0.93,0]$} & -1 & {$[-3.599,-2.666]$} & 4 \\
\hline
\end{tabular}

A necessary and sufficient condition for the stability of the equilibrium points $X_{+}^{*}$ and $X_{-}^{*}$ is given by the condition:

$$
\begin{aligned}
& \beta+\cos \theta+\alpha \sin \theta>0 \\
& 2 \alpha \cos \theta[\beta(\gamma-1) \sin \theta-p]>0 \\
& -2 \alpha \cos \theta[\beta(\gamma-1) \sin \theta-p]+(\beta+\cos \theta+\alpha \sin \theta) \\
& \quad \cdot\{\beta(\cos \theta+\alpha \sin \theta)+2 \sin \theta \cos \theta[\beta(\gamma-1) \sin \theta-p]\}>0
\end{aligned}
$$

This time, we want to have chaotic attractors on the state space between the limits $x_{a}$ and $x_{b}$ for an $x$-axis projection:

$$
\text { on the } x \text {-axis: } \quad x \in\left[x_{a} ; x_{b}\right] \text {. }
$$

The state space domain for an $y$-axis projection is:

$$
\text { on the } y \text {-axis: } y \in\left[x_{a} \frac{\sin \theta}{\cos \theta} ; x_{b} \frac{\sin \theta}{\cos \theta}\right] \text {. }
$$

Substituting Eq. (49) into Eq. (38), the anticontrol switching piecewise-constant controller $p$ has the following form:

$$
\text { controller: } \quad p \in\left[\beta(\gamma-1) \sin \theta-\frac{x_{b}^{2}}{\cos ^{2} \theta} ; \beta(\gamma-1) \sin \theta-\frac{x_{a}^{2}}{\cos ^{2} \theta}\right] \text {. }
$$

The independent attractors are generated on a line, according to Eq. (36), with $\theta$ rotation degree reported to the $x-y$ state axes. As Eq. (37) shows, $z$ state variable depends only on the $\gamma$ and $\theta$ parameters, and not on $x$ or $y$. In order to generate attractors on a plane where $z$ is constant, $\gamma$ takes the following expression:

$$
\gamma=\frac{z}{\sin \theta}+1
$$

for different values of $\theta$. The attractors number is:

$$
N_{\text {max }}=\left[\frac{\sigma\left|x_{b}-x_{a}\right|}{2 \pi}\right]+1
$$

For the numerical simulation, let us assume, as in the system (14), that $\alpha=10$ and the independent periodic attractors are generated on the plains $z=1$ and $z=-1$. The equilibrium points $X_{+}^{*}$ and $X_{-}^{*}$ are stable. Table 2 first presents limits imposed to the state space domain for an $x\left(x_{a}\right.$ and $\left.x_{b}\right)$-axis projection, then the state space domain for an $y$-axis projection calculated with Eq. (50), and the switch levels of the anticontrol switching piecewise-constant controller $p$ of Eq. (51) and finally the maximum number of chaotic attractors (Eq. (53)) which can be generated (Fig. 14).

For the six cases illustrated, the attractors projections onto $x-y$ planes of Fig. 15 show that all the attractors are situated inside the state space given by Table 2 (columns 9 and 10). On the other hand, there is a difference of one attractor between the numerical simulation of the independent chaotic attractors number generated on lines 4 and 5 and the maximum number of attractors given by the mathematical formula of Eq. (53). These two cases correspond to the first case of attractors repartition inside the state space domain $\left[x_{a} ; x_{b}\right]$ is available, as in Fig. 4 . For the other state space domains $\left[x_{a} ; x_{b}\right]$, the theoretical and simulated attractors number coincide. 


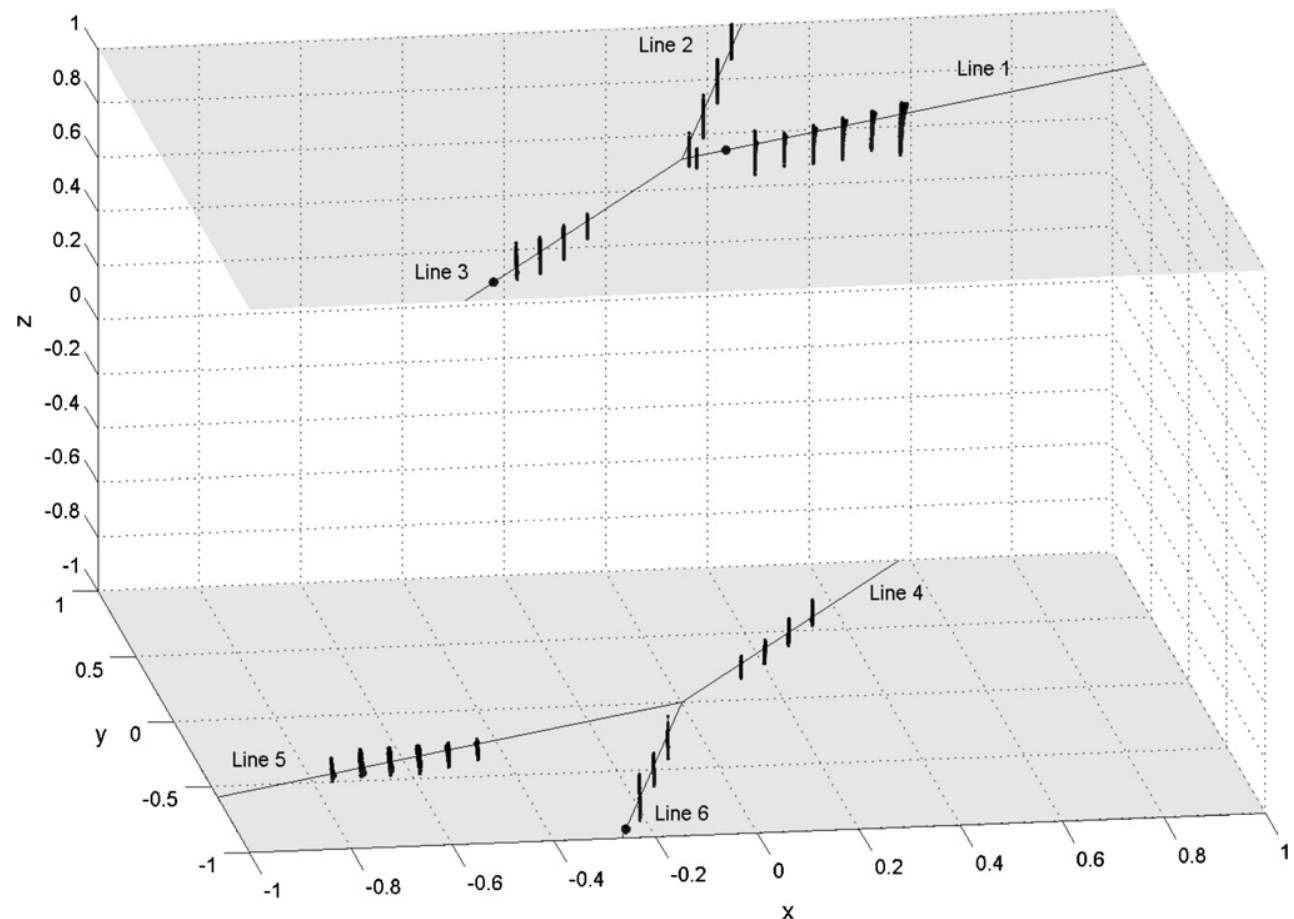

Fig. 14. The independent chaotic attractors generation of the system (33) on the lines 1,2 and 3 of the first plane. The independent chaotic attractors generation of the system (33) on the lines 4,5 and 6 of the second plane.
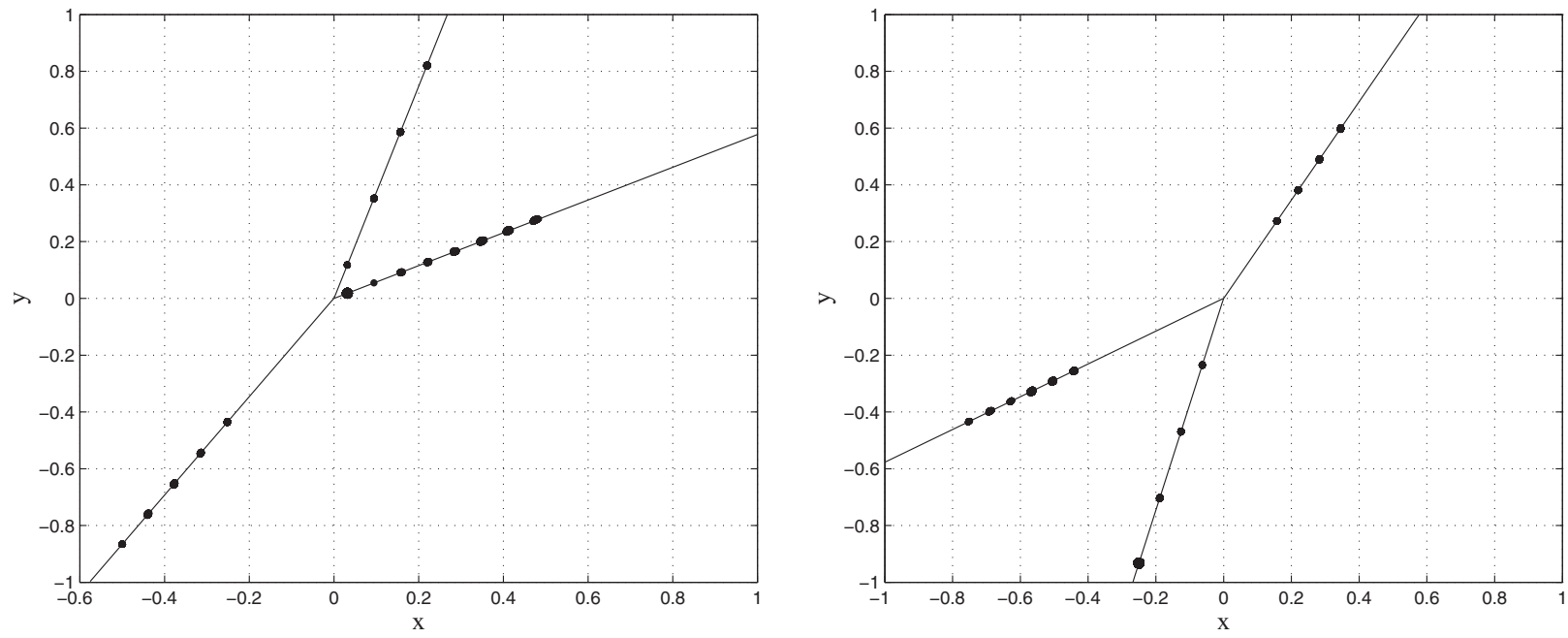

Fig. 15. The projection onto $x-y$ plane of the lines 1 (with the theoretical state space domain $x \in[0,0.5], y \in[0,0.28]$ and $z=1), 2(x \in[0,0.25]$, $y \in[0,0.933]$ and $z=1), 3(x \in[-0.5,-0.2], y \in[-0.87,-0.35]$ and $z=1)$ and $4(x \in[0.1,0.4], y \in[0.17,0.69]$ and $z=-1), 5(x \in[-0.8,-0.4]$, $y \in[-0.46,-0.23]$ and $z=-1), 6(x \in[-0.25,0], y \in[-0.93,0]$ and $z=-1)$.

\section{Conclusion}

This paper introduces a new technique to generate independent chaotic attractors using a switching piecewiseconstant controller in continuous-time nonchaotic systems (with an equilibrium point or a limit cycle). We demonstrate that the equidistant repartition of these attractors is in a precise zone (a controllable state space area) of a precise curve, which depends of the system parameters. We determine the state space domains where the attractors are generated 
from different initial conditions. A mathematical formula giving the maximal attractors number in function of the controller piecewise-constant values is then deduced. A control engineering application can be to make a nonlinear system converge to some attractors of interest, starting from different initial conditions, in order to reach different regimes of operation.

\section{References}

[1] G. Alvarez, F. Montoya, M. Romera, G. Pastor, Breaking two secure communication systems based on chaotic masking, IEEE Trans. Circ. Syst. 51 (2004) 505-506.

[2] J. Awrejcewicz, C. Lamarque, Bifurcation and Chaos in Nonsmooth Mechanical Systems, World Scientific Publishing Co., Singapore, 2003.

[3] M.-F. Danca, K. Wallace, S. Tang, G. Chen, A switching scheme for synthesizing attractors of dissipative chaotic systems, Appl. Math. Comput. 201 (2008) 650-667.

[4] J. Deane, P. Ashwin, D. Hamill, D. Jefferies, Calculation of the periodic spectral components in a chaotic dc-dc converter, IEEE Trans. Circ. Syst. 46 (1999) 1313-1319.

[5] M. diBernardo, C.J. Budd, A. Champneys, P. Kowalczyk, Piecewise-Smooth Dynamical Systems: Theory and Applications, vol. 163 of Appl. Math. Scien., Springer, London, 2008.

[6] L. Endersen, N. Skarland, Limit cycle oscillations in pacemaker cells, IEEE Trans. Biomed. Eng. 47 (2000) 1134-1137.

[7] M. Feckan, Topological Degree Approach to Bifurcation Problems, Springer, 2008.

[8] H. Fujisakaa, T. Yamadab, G. Kinoshitaa, T. Konoa, Chaotic phase synchronization and phase diffusion, Physica D 205 (2006) $41-47$.

[9] M. Kennedy, Three steps to chaos. Part I. Evolution, IEEE Trans. Circ. Syst. 40 (1993) 640-656.

[10] Z. Li, J. Park, G. Chen, H. Young, Y. Choi, Generating chaos via feedback control from a stable ts fuzzy system through a sinusoidal nonlinearity, Int. J. Bifurcat. Chaos 12 (2002) 2283-2291.

[11] J. Lü, X. Yu, G. Chen, Generating chaotic attractors with multiple merged basins of attraction: a switching piecewise-linear control approach, IEEE Trans. Circ. Syst. 50 (2003) 198-207.

[12] J. Lü, X. Yu, T. Zhou, G. Chen, X. Yang, Generating chaos with a switching piecewise-linear controller, Chaos 12 (2002) $344-349$.

[13] R. Lukac, K. Plataniotis, Bit-level based secret sharing for image encryption, Pattern Recogn. 38 (2005) 767-772.

[14] G. Mahmoud, S. Aly, M. Al-Kashif, Dynamical properties and chaos synchronization of a new chaotic complex nonlinear system, Nonlinear Dynam. 51 (2006) 171-181.

[15] C. Morel, M. Bourcerie, F. Chapeau-Blondeau, Generating independent chaotic attractors by chaos anticontrol in nonlinear circuits, Chaos Soliton Fract. 26 (2005) 541-549.

[16] C. Morel, M. Bourcerie, F. Chapeau-Blondeau, Improvement of power supply electromagnetic compatibility by extension of chaos anticontrol, J. Circ. Syst. Comp. 14 (2005) 757-770.

[17] C. Morel, R. Vlad, E. Chauveau, A new technique to generate independent periodic attractors in the state space of nonlinear dynamic systems, Nonlinear Dynam. 59 (2009) 45-60.

[18] C. Morel, R. Vlad, J.-Y. Morel, Anticontrol of chaos reduces spectral emissions, J. Comput. Nonlinear Dynam. 3 (2008) 041009-1-041009-6.

[19] M. Peng, Symmetry breaking, bifurcations, periodicity and chaos in the Euler method for a class of delay differential equations, Chaos Soliton Fract. 24 (2005) 1287-1297.

[20] M. Peng, A. Ucar, The use of the Euler method in identification of multiple bifurcations and chaotic behavior in numerical approximations of delay differential equations, Chaos Soliton Fract. 21 (2004) 883-891.

[21] M.S. Peng, Bifurcation and chaotic behavior in the Euler method for a Kaplan-Yorke prototype delay model, Chaos Soliton Fract. 20 (2004) 489-496.

[22] W. Tang, G. Zhong, G. Chen, K. Man, Generation of N-scroll attractors via sine function, IEEE Trans. Circ. Syst. 48 (2001) $1369-1372$.

[23] T. Ueta, G. Chen, Bifurcation analysis of Chen's equation, Int. J. Bifurcat. Chaos 10 (2000) 1917-1931.

[24] X. Wang, G. Chen, X. Yu, Anticontrol of chaos in continuous-time systems via time-delay feedback, Chaos 10 (2000) 771-779.

[25] J. Warminski, G. Litak, K. Szabelski, Synchronisation and chaos in a parametrically and self-excited system with two degrees of freedom, Nonlinear Dynam. 22 (2000) 125-143.

[26] S. Yu, J. Lü, H. Leung, G. Chen, Design and implementation of n-scroll chaotic attractors from a general jerk circuit, IEEE Trans. Circ. Syst. 52 (2005) 1459-1476. 